\title{
Fixed point theorems for self and non-self F-contractions in metric spaces endowed with a graph
}

\author{
Awais Younus*, Muhammad Umer Azam and Muhammad Asif
}

*Correspondence:

awais@bzu.edu.pk

CASPAM, Bahauddin Zakariya

University, Multan, Pakistan

\begin{abstract}
The main results obtained in this paper are fixed point theorems for self and non-self $G$ F-contractions on metric spaces endowed with a graph. Our new results are generalization of recently fixed point theorems for self mappings on metric spaces and also fixed point theorems for non-self mappings in Banach spaces by using the concept of new type of contractive mappings namely F-contractions.
\end{abstract}

Keywords: Fixed point, F-contraction, Non-self G F-contraction, Directed graph, $G$ $F$-contraction, Non-self mapping, Rothe's boundary condition

2010 AMS Subject Classifications: 47H10, 54H25, 47J26, 54E50, 05C40

\section{Introduction}

The well-known Banach contraction theorem [1] has plenty of extensions in the literature (see, for example, $[2,3]$ ). That theorem states that every self-mapping $f$ defined on complete metric space $(S, d)$ satisfying

$$
d(f r, f s) \leq \alpha d(r, s) \forall r, s \in S,
$$

where $\alpha \in(0,1)$ has a unique fixed point, i.e., there exists a unique $r^{*} \in S$ such that $f r^{*}=r^{*}$.

The extension of Banach contraction theorem for non-self multi-valued mappings was first studied by Assad and Kirk [4] in 1972. After this initiation, lot of fixed point theorems for non-self mappings have been proved by various authors, see, for example, [5-7] and [8].

Firstly, the study of fixed point theorem for single-valued monotone mappings in a metric space endowed with a partial ordering has been investigated by Ran and Reurings [9] and presented its applications to matrix equations. After this, many results in this direction were studied by different authors; see [10-12] and [13]. These theorems are actually hybrids of two fundamental theorems of fixed point theory: the Kanaster-Tarski theorem [14] and the Banach Contraction Principle. Jachymski [15] established the fixed point theorems by using graphs which is the generalization of concept of partial ordering in

(c) The Author(s). 2020 Open Access This article is licensed under a Creative Commons Attribution 4.0 International License, which permits use, sharing, adaptation, distribution and reproduction in any medium or format, as long as you give appropriate credit to the original author(s) and the source, provide a link to the Creative Commons licence, and indicate if changes were made. The images or other third party material in this article are included in the article's Creative Commons licence, unless indicated otherwise in a credit line to the material. If material is not included in the article's Creative Commons licence and your intended use is not permitted by statutory regulation or exceeds the permitted use, you will need to obtain permission directly from the copyright holder. To view a copy of this licence, visit http://creativecommons.org/licenses/by/4.0/. 
metric spaces. Jachymski [15, Theorem 3.2] generalized the Banach contraction theorem for self-mappings on complete metric spaces endowed with the graph, where as Berinde [16, Theorem 3.1] for non-self mappings to Banach spaces endowed with a graph by using the inwardness condition defined in [17]. There are few other fixed point theorems for non-self mappings to Banach spaces endowed with a graph , see, for example, [18] and [19].

Recently, Wardowski [20] introduced a new type of contraction by using a particular function $F: \mathbb{R}^{+} \rightarrow \mathbb{R}$ called $F$-contraction and gave examples to show the validity of such extensions in complete metric spaces. The author proved a new fixed point theorem by using this concept of $F$-contraction.

This paper has been organized in the following manner: In the "Preliminaries" section, we will give the brief introduction of a new type of contraction called $F$-contraction. In the last section, we present a few preliminary notations and our main aim is to study the fixed point theorems for self-mappings as well as non-self mappings using $F$-contractions for metric spaces endowed with a graph. These theorems are the generalization of fixed point theorems discussed by Berinde [16] on Banach spaces endowed with a graph and Wardowski [20] on complete metric spaces.

\section{Preliminaries}

In this section, we present some definitions, examples and results from [20], which will be used in this article. Throughout this paper, consider $\mathbb{R}$ be the set of all real numbers, $\mathbb{R}^{+}$be the set of all positive real numbers and $\mathbb{N}$ be the set of all positive integers.

Definition 1 Let the mapping $F: \mathbb{R}^{+} \rightarrow \mathbb{R}$ satisfies the following conditions:

(f1) $\quad F$ is strictly increasing;

(f2) for each sequence $\left\{r_{n}\right\} \subset \mathbb{R}^{+} \lim _{n \rightarrow+\infty} r_{n}=0$ iff $\lim _{n \rightarrow+\infty} F\left(r_{n}\right)=-\infty$;

(f3) there exists $k \in(0,1)$ provided that $\lim _{\lambda \rightarrow 0^{+}} \lambda^{k} F(\lambda)=0$.

The collection of all such mappings is denoted by $\Omega$.

Definition 2 Let $(S, d)$ be a metric space. A mapping $\Upsilon: S \rightarrow S$ is said to be F-contraction if there exist $F \in \Omega$ and $\tau>0$ provided that

$$
d(\Upsilon s, \Upsilon r)>0 \Longrightarrow \tau+F(d(\Upsilon s, \Upsilon r)) \leq F(d(s, r))
$$

for all $r, s \in S$.

Example 1 Let $F \in \Omega$ be defined by $F(\alpha)=\ln \alpha$. For any $k \in(0,1)$, it is clear that every mapping $\Upsilon: S \rightarrow S$ satisfying (2) is an F-contraction such that

$$
d(\Upsilon s, \Upsilon r) \leq e^{-\tau} d(r, s) \forall r, s \in S, \Upsilon r \neq \Upsilon s .
$$

Example 2 Consider $F \in \Omega$ be defined by $F(\alpha)=\frac{-1}{\sqrt{\alpha}}, \alpha>0$. In this case, for any $k \in(1 / 2,1)$, every F-contraction $\Upsilon$ satisfies

$$
d(\Upsilon r, \Upsilon s) \leq \frac{1}{(1+\tau \sqrt{d(r, s)})^{2}} d(r, s) \forall r, s \in S, \Upsilon r \neq \Upsilon s .
$$


Wardowski stated the $F$-contraction theorem for self mappings in complete metric spaces as follows.

Theorem 1 Let a mapping $T: S \rightarrow S$ be an F-contraction and $(S, d)$ be a complete metric space. Then, $T$ has a unique fixed point $s^{*} \in S$ and for every $s \in S$ the sequence $\left(T^{n} s\right)_{n \in \mathbb{N}}$ converges to $s^{*}$.

Remark 1 From (f1) and (2), we can conclude that every F-contraction $\Upsilon$ is a contractive mappping, i.e.,

$$
d(\Upsilon r, \Upsilon s) \leq d(r, s) \text { for all } r, s \in S \text { and } \Upsilon r \neq \Upsilon s .
$$

Thus, every F-contraction is continuous mapping.

\section{Fixed point theorems in metric spaces endowed with a graph}

By using the concept of $F$-contractions, we establish fixed point theorems for self as well as non-self mappings in complete metric spaces endowed with a graph.

Some graph theory terminologies will be presented here. Let $(S, d)$ be metric space and $\triangle$ denote the diagonal of Cartesian product $S \times S$. Let $G=(V(G), E(G))$ be a directed graph such that $E(G)$, the set of its edges consists of all loops, that is, $\triangle \subset E(G)$ and $V(G)$, the vertex set coincides with $S$. Let $G$ has no parallel edges (arcs). For more details of these terminologies and notations see [21] and [22].

$G^{-1}$ is the converse graph of $G$, i.e., the edge set of $G^{-1}$ is obtained by reversing the edges of $G$, defined as:

$$
E\left(G^{-1}\right)=\{(r, s) \in S \times S:(s, r) \in E(G)\} .
$$

If $s, r$ are vertices in the graph $G$, then a path from $s$ to $r$ of length $t$ is a sequence $\left\{s_{i}\right\}_{i=1}^{t}$ of $t+1$ vertices of $G$ such that $s_{0}=s, s_{t}=r$ and $\left(s_{i-1}, s_{i}\right) \in E(G), i=1,2 \cdots t$.

A graph $G$ is called connected if there exist at least a path between two arbitrary vertices. If $\tilde{G}=(S, E(\tilde{G}))$ is the symmetric graph obtained by placing together the vertices of both $G$ and $G^{-1}$, that is,

$$
E(\tilde{G})=E(G) \cup E\left(G^{-1}\right),
$$

then $G$ is said to be weakly connected whenever $\tilde{G}$ is connected.

$$
\text { If } G=(V(G), E(G)) \text { is a graph and } V(G) \supset H \text {, then the graph }(H, E(G)) \text { with }
$$

$$
E(H)=E(G) \cap(H \times H)
$$

is said to be the subgraph of $G$ determined by $H$, denoted by $G_{H}$.

\section{Self $F$-contraction case}

A mapping $\Upsilon: S \rightarrow S$ is said to be defined on a metric space endowed with a graph $G$ if it satisfies

$$
\forall r, s \in S, \quad(r, s) \in E(G) \text { implies }(\Upsilon r, \Upsilon s) \in E(G) .
$$

A mapping $\Upsilon: S \rightarrow S$ defined on metric space endowed with a graph $G$, is said to be a $G$ $F$-contraction, if there is a constant $\tau>0$ such that $\forall r, s \in S$ with $(r, s) \in E(G)$, we have

$$
[d(\Upsilon r, \Upsilon s)>0 \Longrightarrow \tau+F(d(\Upsilon r, \Upsilon s)) \leq F(d(r, s))]
$$


If $\Upsilon r=r$, then the element $r \in S$ is said to be the fixed point of mapping $\Upsilon$.

Theorem 2 Suppose $(S, d, G)$ be a complete metric space endowed with a weakly connected and directed graph $G$ such that the following property $(T)$ holds, that is, for any sequence $\left\{r_{n}\right\}_{n=1}^{\infty} \subset S$ with $r_{n} \rightarrow r$ as $n \rightarrow \infty$ and $\left(r_{n}, r_{n+1}\right) \in E(G)$ for all $n \in \mathbb{N}$, there exists a subsequence $\left\{r_{s_{n}}\right\}_{n=1}^{\infty}$ satisfying

$$
\left(r_{s_{n}}, r\right) \in E(G), \forall n \in \mathbb{N} \text {. }
$$

Let $\Upsilon: S \rightarrow S$ be a G F-contraction. If the set

$$
S_{\Upsilon}=\{r \in S:(r, \Upsilon r) \in E(G)\}
$$

is nonempty, then the mapping $\Upsilon$ has a unique fixed point in $S$.

Proof Let $r_{0} \in S_{\Upsilon}$. It follows from (7) that $\left(r_{0}, \Upsilon r_{0}\right) \in E(G)$ and by using (4), we obtain

$$
\left(\Upsilon^{n} r_{0}, \Upsilon^{n+1} r_{0}\right) \in E(G), \forall n \in \mathbb{N} .
$$

Denote $r_{n}:=\Upsilon^{n} r_{0}$ for all $n \in \mathbb{N}$. Then, by the fact that $\Upsilon$ is a $G F$-contraction and in view of (4), we get

$$
F\left(d\left(r_{n}, r_{n+1}\right)\right) \leq F\left(d\left(r_{n-1}, r_{n}\right)\right)-\tau,
$$

for all $n \in \mathbb{N}$. Denote $\alpha_{n}=d\left(r_{n}, r_{n+1}\right), n=0,1, \ldots$

Let $r_{n+1} \neq r_{n}$, for every $n \in \mathbb{N} \cup\{0\}$. Then, $\alpha_{n}>0$ for all $n \in \mathbb{N} \cup\{0\}$ and by using (2), we get

$$
F\left(\alpha_{n}\right) \leq F\left(\alpha_{n-1}\right)-\tau \leq F\left(\alpha_{n-2}\right)-2 \tau \leq \cdots \leq F\left(\alpha_{0}\right)-n \tau .
$$

Hence, $\lim _{n \rightarrow \infty} F\left(\alpha_{n}\right)=-\infty$. By the property (f2), we obtain that $\alpha_{n} \rightarrow 0$ as $n \rightarrow \infty$. From (f3), there exists $k \in(0,1)$ such that $\lim _{n \rightarrow \infty} \alpha_{n}^{k} F\left(\alpha_{n}\right)=0$. By (10), the following holds for all $n \in \mathbb{N}$

$$
\alpha_{n}^{k} F\left(\alpha_{n}\right)-\alpha_{n}^{k} F\left(\alpha_{0}\right) \leq \alpha_{n}^{k}\left(F\left(\alpha_{0}\right)-n \tau\right)-\alpha_{n}^{k} F\left(\alpha_{0}\right)=-\alpha_{n}^{k} n \tau .
$$

Letting $n \rightarrow \infty$ in (11), we deduce $\lim _{n \rightarrow \infty} n \alpha_{n}^{k}=0$. From (11), we observe that there exists $n^{\prime} \in \mathbb{N}$ such that $n \alpha_{n}^{k} \leq 1$ for all $n \geq n^{\prime}$. Consequently, we have

$$
\alpha_{n} \leq \frac{1}{n^{1 / k}} \text { for all } n \geq n^{\prime}
$$

Choose $m, n \in \mathbb{N}$ such that $m \geq n \geq n^{\prime}$ and from (12), we have

$$
d\left(r_{m}, r_{n}\right) \leq \alpha_{m-1}+\cdots+\alpha_{n}<\sum_{j=n}^{\infty} \alpha_{n} \leq \sum_{j=n}^{\infty} \frac{1}{j^{1 / k}} .
$$

The convergence of the series $\sum_{j=n}^{\infty} \frac{1}{j^{1 / k}}$ implies that $\left\{r_{n}\right\}$ is a Cauchy sequence, hence convergent in $(S, d, G)$. The limit of this sequence is denoted as:

$$
\lim _{n \rightarrow \infty} r_{n}=r^{*}
$$

By using property $(\mathrm{T})$ of $(S, d, G)$, there exists a subsequence $\left\{r_{s_{n}}\right\}$ satisfying

$$
\left(r_{s_{n}}, r^{*}\right) \in E(G), \forall n \in \mathbb{N} \text {. }
$$

Hence, by inequality (5) and in view of (4), we get

$$
F\left(d\left(\Upsilon r_{s_{n}}, \Upsilon r^{*}\right)\right) \leq F d\left(r_{s_{n}}, r^{*}\right)-\tau<F\left(d\left(r_{s_{n}}, r^{*}\right)\right),
$$


which implies

$$
d\left(\Upsilon r_{s_{n}}, \Upsilon r^{*}\right) \leq d\left(r_{s_{n}}, r^{*}\right)
$$

Therefore, by triangle inequality, we have

$$
\begin{aligned}
d\left(r^{*}, \Upsilon r^{*}\right) & \leq d\left(r^{*}, r_{s_{n}+1}\right)+d\left(r_{s_{n}+1}, \Upsilon r^{*}\right) \\
& =d\left(r^{*}, r_{s_{n}+1}\right)+d\left(\Upsilon r_{s_{n}}, \Upsilon r^{*}\right)
\end{aligned}
$$

By using (15), inequality (16) yields

$$
d\left(r^{*}, \Upsilon r^{*}\right) \leq d\left(r^{*}, r_{s_{n}+1}\right)+d\left(r_{s_{n}}, r^{*}\right),
$$

for all $n \geq 1$. In Eq. (17), assuming $n \rightarrow \infty$ and using (13), we have $d\left(r^{*}, \Upsilon r^{*}\right)=0$, which implies $r^{*}=\Upsilon r^{*}$, i.e., $r^{*}$ a fixed point of mapping $\Upsilon$.

Note that the uniqueness of $r^{*}$ follows by the G F-contraction condition (5).

Remark If we use the mapping $F \in \Omega$ defined by the formula $F(\alpha)=\ln \alpha$ in Theorem 2, then for all $k \in(0,1)$, we obtain the extension of [16, Theorem 2.1].

Example Let $(S, d)$ be the complete metric space and $G$ be the complete graph on the set $S$, that is, $E(G)=S \times S$. Let the mapping $F \in \Omega$ be defined as: $F(\alpha)=\ln \alpha$, then the $G$ F-contraction (5) is actually a F-contraction (2) which reduces to Banach contraction, i.e.,

$$
d(\Upsilon r, \Upsilon s) \leq e^{-\tau} d(\Upsilon r, \Upsilon s), \text { for all } r, s \in S, \Upsilon r \neq \Upsilon s
$$

for any $k \in(0,1)$ and $\tau>0$.

\section{Non-self $F$-contraction case}

Let $S$ be a Banach space, $A$ be a nonempty, closed subset of $S$ and $\Upsilon: A \rightarrow S$ be a non-self mapping. We choose $r \in A$ such that $\Upsilon r \notin A$, then there is an element $s \in \partial A$ such that

$$
s=(1-\mu) r+\mu \Upsilon r \text { where } \mu \in(0,1),
$$

which represents the fact that

$$
d(r, \Upsilon r)=d(r, s)+d(s, \Upsilon r), s \in \partial A
$$

where $d(r, s)=\|r-s\|$.

Caristi [17] used a condition related to (18), called inward condition, to get the generalization of Banach contraction theorem for non-self mappings. The inward condition is more general because it does not need $s$ in (18) to belong to $\partial A$.

A non-self mapping $\Upsilon: A \rightarrow S$ is said to be defined on the Banach space $S$ endowed with a graph $G$, if it satisfies the property that

$$
\begin{aligned}
& \text { for all } r, s \in A(r, s) \in E(G) \\
& \text { with } \Upsilon r, \Upsilon s \in A \text {, implies }(\Upsilon r, \Upsilon s) \in E(G) \cap(A \times A) \text {, }
\end{aligned}
$$

for the subgraph of $G$ induced by $A$.

Theorem 3 Suppose $(S, d, G)$ be a Banach space endowed with a weakly connected and directed graph $G$ provided that following property $(T)$ holds, that is, for any sequence $\left\{r_{n}\right\} \subset$ $S$ along with $r_{n} \rightarrow r$ as $n \rightarrow \infty$ and

$$
\left(r_{n}, r_{n+1}\right) \in E(G), \forall n \in \mathbb{N},
$$


there exists a subsequence $\left\{r_{s_{n}}\right\}$ satisfying

$$
\left(r_{s_{n}}, r\right) \in E(G), \forall n \in \mathbb{N} \text {. }
$$

Let $A$ be a nonempty, closed subset of $S$ and $\Upsilon: A \rightarrow S$ be a $G_{A} F$-contraction, that is, there exists a constant $\tau>0$ such that

$$
\tau+F(d(\Upsilon r, \Upsilon s)) \leq F(d(r, s)) \text { for all }(r, s) \in E\left(G_{A}\right),
$$

where $G_{A}$ is the subgraph of $G$ determined by $A$. If the set

$$
A_{\Upsilon}:=\{r \in \partial A:(r, \Upsilon r) \in E(G)\}
$$

is nonempty and $\Upsilon$ satisfies Rothe's boundary condition

$$
\Upsilon(\partial A) \subset A,
$$

then the mapping $\Upsilon$ has a unique fixed point.

Proof If $\Upsilon(A) \subset A$, then $\Upsilon$ is a self-map of the closed set $A$ and the conclusion follows by Theorem 2. Now, we consider the case that $\Upsilon(A) \not \subset A$. Let $r_{0} \in A_{\Upsilon}$. It follows that $\left(r_{0}, \Upsilon r_{0}\right) \in E(G)$ and in view of equation (4), we have

$$
\left(\Upsilon^{n} r_{0}, \Upsilon^{n+1} r_{0}\right) \in E(G), \text { for all } n \in \mathbb{N} \text {. }
$$

Let we denote $r_{n}:=\Upsilon^{n} r_{0}$, for all $n \in \mathbb{N}$. By virtue of (22) $\Upsilon r_{0} \in A$.

Consider $r_{1} \equiv s_{1}=\Upsilon r_{0}$. Let $\Upsilon r_{1} \in A$, set $r_{2} \equiv s_{2}=\Upsilon r_{1}$. If $\Upsilon r_{1} \notin A$, then we can select an element $r_{2} \in \partial A$ on the segment $\left[r_{1}, \Upsilon r_{1}\right]$, that is,

$$
r_{2}=(1-\mu) r_{1}+\mu \Upsilon r_{1} \text {, where } \mu \in(0,1) \text {. }
$$

By following the same method, we obtain two sequences $\left\{r_{n}\right\}$ and $\left\{s_{n}\right\}$ whose terms satisfy one of the succeeding properties:

(i) $r_{n} \equiv s_{n}=\Upsilon r_{n-1}$, if $\Upsilon r_{n-1} \in A$;

(ii) $\quad r_{n}=(1-\mu) r_{n-1}+\mu \Upsilon r_{n-1} \in \partial A, \mu \in(0,1), \Upsilon r_{n-1} \notin A$.

For the simplicity of arguments in the proof, let us denote

$$
U=\left\{r_{a} \in\left\{r_{n}\right\}: r_{a}=s_{a}=\Upsilon r_{a-1}\right\}
$$

and

$$
Z=\left\{r_{a} \in\left\{r_{n}\right\}: r_{a} \neq \Upsilon r_{a-1}\right\}
$$

Note that $\left\{r_{n}\right\} \subset A$ for all $n \in \mathbb{N}$. Moreover, if $r_{a} \in Z$, then both $r_{a-1}$ and $r_{a+1}$ belong to set $U$. The sequence $\left\{r_{n}\right\}$ can have consecutive terms $r_{a}$ and $r_{a+1}$ in set $U$, but this assertion is not true for the set $Z$. First of all we have to prove that

$$
r_{a} \neq \Upsilon r_{a-1} \text { implies } r_{a-1}=\Upsilon r_{a-2}
$$

Suppose contrary that $r_{a-1} \neq \Upsilon r_{a-2}$ then $r_{a-1} \in \partial A$. Since $\Upsilon(\partial A) \subset A$ then $\Upsilon r_{a-1} \in A$. Hence, $r_{a}=\Upsilon r_{a-1}$ which is a contradiction.

Here, we have three different cases to show that $\left\{r_{n}\right\}$ is Cauchy sequence which are following:

Case 1. $r_{n}, r_{n+1} \in U$. 
Since both elements belong to set $U$, therefore, we have $r_{n}=s_{n}=\Upsilon r_{n-1}$ and $r_{n+1}=$ $s_{n+1}=\Upsilon r_{n}$. Hence,

$$
d\left(r_{n+1}, r_{n}\right)=d\left(s_{n+1}, s_{n}\right)=d\left(\Upsilon s_{n}, \Upsilon s_{n-1}\right),
$$

where $\left(s_{n}, s_{n-1}\right) \in E(G)$ by virtue of (23). Therefore, we have

$$
d\left(\Upsilon s_{n}, \Upsilon s_{n-1}\right)=d\left(\Upsilon r_{n}, \Upsilon r_{n-1}\right)>0 .
$$

Consequently, we get the following inequality

$$
\tau+F\left(d\left(\Upsilon s_{n}, \Upsilon s_{n-1}\right)\right) \leq F\left(d\left(s_{n}, s_{n-1}\right)\right),
$$

by using (21).

Case 2. $r_{n} \in U, r_{n+1} \in Z$.

In this case, we have $r_{n}=s_{n}=\Upsilon r_{n-1}$, but $r_{n+1} \neq s_{n+1}=\Upsilon r_{n}$; therefore, we have

$$
d\left(r_{n}, \Upsilon r_{n}\right)=d\left(r_{n}, r_{n+1}\right)+d\left(r_{n+1}, \Upsilon r_{n}\right) .
$$

The above equality implies $d\left(r_{n+1}, \Upsilon r_{n}\right) \neq 0$ and hence

$$
d\left(r_{n}, r_{n+1}\right)=d\left(r_{n}, \Upsilon r_{n}\right)-d\left(r_{n+1}, \Upsilon r_{n}\right)<d\left(r_{n}, \Upsilon r_{n}\right)=d\left(\Upsilon r_{n-1}, \Upsilon r_{n}\right),
$$

since $r_{n} \in U$. By using (25), we obtain

$$
d\left(r_{n}, r_{n+1}\right)<d\left(\Upsilon r_{n-1}, \Upsilon r_{n}\right)=d\left(\Upsilon s_{n-1}, \Upsilon s_{n}\right)>0 .
$$

We can obtain again inequality (24) by using the similar arguments to that in case 1 .

Case 3. $r_{n} \in Z, r_{n+1} \in U$.

In this case, we have $r_{n+1}=\Upsilon r_{n}$, and $r_{n} \neq s_{n}=\Upsilon r_{n-1}$. Since $r_{n} \in Z$, so we have

$$
d\left(r_{n-1}, \Upsilon r_{n-1}\right)=d\left(r_{n-1}, r_{n}\right)+d\left(r_{n}, \Upsilon r_{n-1}\right) .
$$

Hence, by triangle inequality

$$
\begin{aligned}
d\left(r_{n}, r_{n+1}\right) & \leq d\left(r_{n}, \Upsilon r_{n-1}\right)+d\left(\Upsilon r_{n-1}, r_{n+1}\right) \\
& =d\left(r_{n}, \Upsilon r_{n-1}\right)+d\left(\Upsilon r_{n-1}, \Upsilon r_{n}\right) \\
& =d\left(r_{n}, \Upsilon r_{n-1}\right)+d\left(\Upsilon s_{n-1}, \Upsilon s_{n}\right) .
\end{aligned}
$$

By virtue of $(23)\left(s_{n-1}, s_{n}\right) \in E(G)$, and the following inequality is obtained by the contraction condition (21)

$$
F\left(d\left(\Upsilon s_{n-1}, \Upsilon s_{n}\right)\right) \leq F\left(d\left(s_{n-1}, s_{n}\right)\right)-\tau<F\left(d\left(s_{n-1}, s_{n}\right)\right),
$$

which implies

$$
d\left(\Upsilon s_{n-1}, \Upsilon s_{n}\right) \leq d\left(s_{n-1}, s_{n}\right)=d\left(r_{n-1}, r_{n}\right) .
$$

Thus, by using (26) and (29) in inequality (27), we have

$$
\begin{aligned}
d\left(r_{n}, r_{n+1}\right) & \leq d\left(r_{n}, \Upsilon r_{n-1}\right)+d\left(\Upsilon s_{n-1}, \Upsilon_{s_{n}}\right) \\
& <d\left(r_{n}, \Upsilon r_{n-1}\right)+d\left(r_{n-1}, r_{n}\right) \\
& =d\left(r_{n-1}, \Upsilon r_{n-1}\right)
\end{aligned}
$$

By using $(23),\left(r_{n-2}, r_{n-1}\right)=\left(s_{n-2}, s_{n-1}\right) \in E(G)$ and by virtue of contraction condition (21), we get

$$
d\left(r_{n}, r_{n+1}\right)<d\left(r_{n-1}, \Upsilon r_{n-1}\right)=d\left(\Upsilon r_{n-2}, \Upsilon r_{n-1}\right) \leq d\left(r_{n-2}, r_{n-1}\right) .
$$


Now, we summarize all the above mentioned three cases. By virtue of (24) and (30), it follows that the sequence $\left\{d\left(r_{n}, r_{n+1}\right)\right\}$ satisfies the inequality

$$
\tau+F\left(\max \left\{d\left(r_{n-2}, r_{n-1}\right), d\left(r_{n-1}, r_{n}\right)\right\}\right) \leq F\left(d\left(r_{n}, r_{n+1}\right)\right),
$$

for all $n \geq 2$. Denote $\alpha_{n}=d\left(r_{n}, r_{n+1}\right)$ for $n=2,3, \cdots$.

We obtain the following inequality by simple induction for $n \geq 2$, and using (31)

$$
F\left(\alpha_{n}\right) \leq F\left(\max \left\{\alpha_{0}, \alpha_{1}\right\}\right)-\left[\frac{n}{2}\right] \tau,
$$

where $\left[\frac{n}{2}\right]$ denotes the greatest integer not exceeding $\frac{n}{2}$.

Hence, $\lim _{n \rightarrow \infty} F\left(\alpha_{n}\right)=-\infty$. By the property (f2), we obtain that $\alpha_{n} \rightarrow 0$ as $n \rightarrow \infty$. From (f3), there exists $k \in(0,1)$ such that $\lim _{n \rightarrow \infty} \alpha_{n}^{k} F\left(\alpha_{n}\right)=0$. Denote $\gamma=\max \left\{\alpha_{0}, \alpha_{1}\right\}$. By (32), the following holds for all $n \geq 2$ :

$$
\alpha_{n}^{k} F\left(\alpha_{n}\right)-\alpha_{n}^{k} F(\gamma) \leq \alpha_{n}^{k}\left(F(\gamma)-\left[\frac{n}{2}\right] \tau\right)-\alpha_{n}^{k} F(\gamma)=-\alpha_{n}^{k}\left[\frac{n}{2}\right] \tau .
$$

Assuming $n \rightarrow \infty$ in (33), we deduce $\lim _{n \rightarrow \infty}\left[\frac{n}{2}\right] \alpha_{n}^{k}=0$. From (33), we observe that there exists $n^{\prime} \in \mathbb{N}$ such that $\left[\frac{n}{2}\right] \alpha_{n}^{k}<n \alpha_{n}^{k} \leq 1$ for all $n \geq n^{\prime}$. Consequently, we have

$$
\alpha_{n} \leq \frac{1}{n^{1 / k}} \text { for all } n \geq n^{\prime} .
$$

Choose $m, n \in \mathbb{N}$ such that $m \geq n \geq n^{\prime}$ and from (34), we have

$$
d\left(r_{m}, r_{n}\right) \leq \alpha_{m-1}+\cdots+\alpha_{n}<\sum_{j=n}^{\infty} \alpha_{n} \leq \sum_{j=n}^{\infty} \frac{1}{j^{1 / k}}
$$

The convergence of the series $\sum_{j=n}^{\infty} \frac{1}{j^{1 / k}}$ implies that $\left\{r_{n}\right\}$ is a Cauchy sequence, hence convergent in $(S, d, G)$. Since $\left\{r_{n}\right\} \subset A$ and $A$ is closed, $\left\{r_{n}\right\}$ converges to some point $r^{\prime} \in A$, i.e., $\lim _{n \rightarrow \infty} r_{n}=r^{\prime}$.

By property $(\mathrm{T})$, there exists a subsequence $\left\{r_{s_{n}}\right\}$ satisfying

$$
\left(r_{s_{n}}, r^{\prime}\right) \in E(G) \text {, for all } n \in \mathbb{N} \text {. }
$$

Hence, by the $F$-contraction condition (21), we get

$$
d\left(\Upsilon r_{s_{n}}, \Upsilon r^{\prime}\right) \leq d\left(r_{s_{n}}, r^{\prime}\right)
$$

Therefore, by triangle inequality, we have

$$
\begin{aligned}
d\left(r^{\prime}, \Upsilon r^{\prime}\right) & \leq d\left(r^{\prime}, r_{s_{n}+1}\right)+d\left(r_{s_{n}+1}, \Upsilon r^{\prime}\right) \\
& =d\left(r^{\prime}, r_{s_{n}+1}\right)+d\left(\Upsilon r_{s_{n}}, \Upsilon r^{\prime}\right) .
\end{aligned}
$$

By using (35), the above inequality yields

$$
d\left(r^{\prime}, \Upsilon r^{\prime}\right) \leq d\left(r^{\prime}, r_{s_{n}+1}\right)+d\left(r_{s_{n}}, r^{\prime}\right)
$$

for all $n \geq 1$. Taking limit $n \rightarrow \infty$ and using (36), we obtain $d\left(r^{\prime}, \Upsilon r^{\prime}\right)=0$ and get $r^{\prime}=\Upsilon r^{\prime}$, which shows that $r^{\prime}$ is a fixed point of $\Upsilon$.

The uniqueness of $r^{*}$ immediately follows by the $G_{A} F$-contraction condition (21).

Remark 3 If we use the mapping $F \in \Omega$ defined by the formula $F(\alpha)=\ln \alpha$ in Theorem 3, then for all $k \in(0,1)$, we obtain the extension of [16, Theorem 3.1]. 
Example 4 Let $S=\mathbb{R}$ be a Banach space with the usual norm and $A=(-\infty, 0]$ is a closed subset of $S$. Let the mapping $\Upsilon: A \rightarrow S$ be defined as:

$$
\Upsilon r= \begin{cases}0 & \text { if } r \in[-1,0] \\ 0.5 & \text { if } r \in(-\infty,-1) .\end{cases}
$$

Let the mapping $F \in \Omega$ be given by the formula $F(\alpha)=\frac{-1}{\sqrt{\alpha}}$, and the edge set of graph $G$ and the subgraph $G_{A}$ determined by $A$ is defined as:

$$
E(G)=\{(r, s) \in S \times S: r \leq s\}
$$

and

$$
E\left(G_{A}\right)=\{(r, s) \in A \times A: r \leq s\},
$$

respectively. It is easy to check that (19) holds, that is,

$$
\text { for all } r, s \in A(r, s) \in E(G)
$$

with $\Upsilon r, \Upsilon s \in A$, implies $(\Upsilon r, \Upsilon s) \in E(G) \cap(A \times A)$.

In view of (19), for $t, u \in(-\infty,-1)$ and $r, s \in[-1,0]$, the edges $(t, u),(t, r)$ has to be removed and for the rest of edges we have

$$
(\Upsilon r, \Upsilon s)=(0,0) \in E\left(G_{A}\right) .
$$

Moreover, $G$ is a weakly connected and for any $k \in(0.5,1), \Upsilon$ is a non-self $G_{A}$ F-contraction on $A$ with $\tau=\frac{1}{\sqrt{d(r, s)}}$, since

$$
d(\Upsilon r, \Upsilon s)=\frac{1}{2}<\frac{1}{4} \times d(r, s) \text { for } r \in(-\infty,-1) \text { and } s \in[-1,0]
$$

(for the rest of edges of $E\left(G_{A}\right)$, the F-contraction condition (21) is obvious, since the quantity in its left hand side is always zero). Property (T) holds with constant sequences $\left\{r_{n}=r\right\}$ satisfying the property $\left(r_{n}, r_{n+1}\right) \in E\left(G_{A}\right)$, for all $n \in \mathbb{N}$. Rothe's boundary condition is also satisfied, as $\partial A=\{0\}$ and so $\Upsilon(\partial A) \subset A$. Finally, since we have $A_{\Upsilon}=\{0\} \neq \emptyset$, all assumptions in Theorem 3 are satisfied and $r^{\prime}=0$ is the fixed point of $\Upsilon$.

\section{Conclusion}

In this paper, we have presented the fixed point theorems for self and non-self $G, F$ contractions on metric spaces endowed with a graph. These theorems immediately imply the extension of recently fixed point theorems for self-mappings on metric spaces and fixed point theorems for non-self mappings in Banach spaces.

Acknowledgements

The authors thank the anonymous reviewers for their careful reading of this paper and their many insightful comments and suggestions.

Authors' contributions

All authors have equally made contributions. The authors read and approved the final manuscript.

Funding

No funding was received.

Availability of data and materials

Not applicable. 
Received: 28 January 2020 Accepted: 27 July 2020

Published online: 01 September 2020

\section{References}

1. Banach, S.: Sur les opérations dans les ensembles abstraits et leur application aux équations intégrales. Fund. Math. 3(1), 133-181 (1922)

2. Soliman, A. H.: Fixed point theorems for a generalized contraction mapping of rational type in symmetric spaces. J. Egypt. Math. Soc. 25(3), 298-301 (2017)

3. Wang, S.: Some fixed point theorems for G-isotone mappings in partially ordered metric spaces. J. Egypt. Math. Soc. 24(3), 410-415 (2016)

4. Assad, N. A., Kirk, W. A.: Fixed point theorems for set-valued mappings of contractive type. Pac. J. Math. 43(3), 553-562 (1972)

5. Alghamdi, M. A., Berinde, V., Shahzad, N. A.: Fixed points of non-self almost contractions. Carpathian J. Math. 30(1), 7-14 (2014)

6. Alghamdi, M. A., Berinde, V., Shahzad, N. A.: Fixed points of multivalued nonself almost contractions. J. Appl. Math. 2013, 6 (2013)

7. Berinde, $\mathrm{V}:$ : A common fixed point theorem for nonself mappings. Miskolc Math. Notes. 5(2), 137-144 (2004)

8. Berinde, $V_{\text {.: }}$ Approximation of fixed points of some nonself generalized $\phi$-contractions. Math. Balkanica. 18(1-2), 85-93 (2004)

9. Ran, A. C., Reurings, M. C.: A fixed point theorem in partially ordered sets and some applications to matrix equations. Proc. Am. Math. Soc. 132(5), 1435-1443 (2004)

10. Nieto, J. J., Rodríguez-López, R.: Contractive mapping theorems in partially ordered sets and applications to ordinary differential equations. Order. 22(3), 223-239 (2005)

11. Bhaskar, T. G, Lakshmikantham, V: Fixed point theorems in partially ordered metric spaces and applications. Nonlinear Anal. 65(7), 1379-1393 (2006)

12. Drici, Z., McRae, F. A., Devi, J. V.: Fixed-point theorems in partially ordered metric spaces for operators with PPF dependence. Nonlinear Anal. 67(2), 641-647 (2007)

13. Beg, I., Butt, A. R.: Fixed point for set-valued mappings satisfying an implicit relation in partially ordered metric spaces. Nonlinear Anal. 71(9), 3699-3704 (2009)

14. Tarski, A.: A lattice theoretical fixed point theorem and its applications. Pacfic J. Math. 5, 285-309 (1955)

15. Jachymski, J.: The contraction principle for mappings on a metric space with a graph. Proc. Am. Math. Soc. 136(4), 1359-1373 (2008)

16. Berinde, V., Pacurar, M.: The contraction principle for nonself mappings on Banach spaces endowed with a graph. J. Nonlinear Convex Anal. 16(9), 1925-1936 (2015)

17. Caristi, J.: Fixed point theorems for mappings satisfying inwardness conditions. Trans. Am. Math. Soc. 215, 241-251 (1976)

18. Balog, L., Berinde, V.: Fixed point theorems for nonself Kannan type contractions in Banach spaces endowed with a graph. Carpathian J. Math. 32(3), 293-302 (2016)

19. Balog, L., Berinde, V., Păcurar, M.: Approximating fixed points of nonself contractive type mappings in banach spaces endowed with a graph. Analele Universitatii" Ovidius" Constanta-Seria Matematica. 24(2), $27-43$ (2016)

20. Wardowski, D.: Fixed points of a new type of contractive mappings in complete metric spaces. Fixed Point Theory Appl. 2012(1), 1-6 (2012)

21. Chartrand, G., Lesniak, L., Zhang, P.: Graphs and digraphs. Chapman and Hall/CRC, Boca Raton (2010)

22. Johnsonbaugh, R.: Essential discrete mathematics. Collier Macmillan, Michigan (1987)

\section{Publisher's Note}

Springer Nature remains neutral with regard to jurisdictional claims in published maps and institutional affiliations.

\section{Submit your manuscript to a SpringerOpen ${ }^{\circ}$ journal and benefit from:}

- Convenient online submission

Rigorous peer review

Open access: articles freely available online

- High visibility within the field

- Retaining the copyright to your article

Submit your next manuscript at $\gg$ springeropen.com 